\title{
Treatment resistant depression
}

\author{
D Ariyasinghe, SS Williams
}

\section{Management of treatment-resistant depression in adolescents (1)}

This study aimed to systematically review the management of treatment-resistant depression in adolescent patients.

The authors conducted an electronic database search of PUBMED, EMBASE, Cochrane, Web of Science and PsycINFO for studies with adolescent treatmentresistant depression published up to January 2014. Treatment-resistant depression was defined as failure to respond to at least one course of psychological or pharmacological treatment for depression with an adequate dosage, duration, and appropriate compliance during the current illness episode. The Cochrane riskof-bias method was used to assess the quality of randomized controlled trials. A meta-analysis of all active treatments was conducted.

Eight studies with 411 depressed adolescents that fit predetermined criteria investigated pharmacological treatments and psychotherapies. Six were open-label studies, and two were randomized controlled trials. The overall response rate for all active treatments investigated was $46 \%$ (95\% CI 33 to 59 ; $\mathrm{N}=411$ ) with a moderately high degree of heterogeneity (I2 $=76.1 \%$, $95 \% \mathrm{CI}=47 \%-86 \%)$. When only the two randomized trials were included, the overall response rate of active treatment was 53\% (95\% CI = 38-67; $\mathrm{N}=347)$. In these randomized trials, SSRI therapy plus CBT was significantly more effective than SSRI therapy alone, while amitriptyline was not more effective than placebo.

The paper concludes that approximately half of the adolescents who presented with treatment-refractory depression responded to active treatment. They recommend the combination of antidepressant medication and psychotherapy for adolescents who present with treatment-resistant depression.

\section{Long-term cognitive and psychological functioning in post-electroconvulsive therapy patients (2)}

Electroconvulsive therapy (ECT) is associated with positive outcomes for treatment-resistant mood disorders in the short term. However, there is limited research on long-term cognitive or psychological changes beyond 1 year after -ECT. This study evaluated long-term outcomes in cognitive functioning, psychiatric symptoms, and quality of life for individuals who had undergone ECT.

Eligible participants ( $\mathrm{N}=294)$ who completed a brief preECT neuropsychological assessment within the last 14 years were recruited for a follow-up evaluation; a limited sample agreed to follow-up testing $(n=34)$. At followup, participants were administered cognitive measures (Repeatable Battery for the Assessment of Neuropsychological Status [RBANS], Wide Range Achievement Test-4 Word Reading, Trail Making Test, Wechsler Adult Intelligence Scale-Fourth Edition Letter Number Sequence and Digit Span, and Controlled Oral Word Association Test), along with emotional functioning measures (Beck Depression Inventory-Second Edition [BDI-II] and Beck Anxiety Inventory) and the World Health Organization Quality of Life-BREF quality of life measure. Follow-up-testing occurred on average (SD) 6.01 (3.5) years after last ECT treatment.

At follow-up, a paired t test showed a large and robust reduction in mean BDI-II score. Scores in cognitive domains remained largely unchanged. A trend was observed for a mean reduction in RBANS visual spatial scores. Lower BDI-II scores were significantly associated with higher RBANS scores and improved quality of life.

The authors conclude that among some of the post-ECT patients included in the study, memory, cognitive functioning, and decreases in depressive symptoms remain intact and stable even several years after ECT. However, the selective sampling at follow-up makes these results difficult to generalize to all post-ECT patients.

\section{Ketamine for treatment resistant depression (3)}

Ketamine is emerging as a hope for patients with treatment resistant depressive disorder. This new study looked at the efficacy, safety, and durability of repeated ketamine infusions for comorbid Posttraumatic Stress Disorder (PTSD) and treatment-resistant depression (TRD).

Individuals with comorbid DSM-5-defined PTSD and DSM-IV-defined major depressive disorder $(\mathrm{N}=15)$ received 6 intravenous ketamine infusions $(0.5 \mathrm{mg} / \mathrm{kg})$ on a Monday-Wednesday-Friday schedule over a 12day period from May 2015 to June 2016. Data from 
outcome measures were collected before and 24 hours after each infusion and weekly for 8 weeks following the final infusion.

Continuous measures of symptom change were significant for both disorders and were associated with large effect sizes (mean decrease in PTSD Checklist for DSM-5 score $=33.3$ points [95\% CI, 23.0-43.5 points], $\mathrm{p}<.0005$, sample size-adjusted Cohen $\mathrm{d}$ [d'] = 2.17; mean decrease in Montgomery-Asberg Depression Rating Scale score $=26.6$ points [ $95 \%$ CI, 23.0-30.2 points], $\left.\mathrm{p}<.0005, \mathrm{~d}^{\prime}=4.64\right)$. The remission rate for PTSD was $80.0 \%$, and the response rate for TRD was 93.3\%.

Participants in remission from PTSD after the infusion series $(\mathrm{n}=12)$ had a median time to relapse of 41 days. Similarly, participants whose depression symptoms responded to the infusion series $(n=14)$ had a median time to relapse of 20 days. Repeated ketamine infusions were associated with transient increases in dissociative symptoms. No participant reported worsening of PTSD symptoms over the study duration.

This study, the first open-label study of repeated ketamine infusions in a comorbid population, found rapid and sustained improvement in PTSD and depression symptoms. This report suggests that repeated ketamine treatments are safe and may represent an efficacious treatment for individuals with comorbid PTSD and TRD.

Psychological therapies for treatmentresistant depression in adults (4)

The first line therapy for moderate to severe depression is pharmacotherapy. However, only one third of these patients will recover completely when treated with antidepressants.

Treatment resistance (TRD) was defined as “A primary diagnosis of unipolar depression that has not responded (or has only partially responded) to a minimum of four weeks of antidepressant treatment (ADT) at a recommended dose (at least $150 \mathrm{mg} / \mathrm{d}$ imipramine or equivalent antidepressant (e.g. $20 \mathrm{mg} / \mathrm{d}$ citalopram).”

Randomized controlled trials (RCTs) and cluster RCTs were included in the review. The patients were in the 1874-year age range. Studies which included patients with co-morbid schizophrenia were excluded but those with co-morbid physical disorders were included.

Studies which included any psychological therapy (psychodynamic/psychoanalytical, cognitivebehavioural, humanistic and integrated therapies) provided either as monotherapy or in combination with ADT for patients with TRD were considered as appropriate interventions.
The primary outcomes were a change in depressive symptoms as measured using clinician rated depressive symptom scales or self rated depressive symptom scales and drop-out rates from the study or treatment. Six studies, which included 698 participants, were included in the meta analysis. A random-effects meta-analysis of five trials $(n=575)$ showed that psychotherapy given in addition to usual care showed improvement in selfreported depressive symptoms (MD -4.07 points, 95\% confidence interval (CI) -7.07 to -1.07 on the Beck Depression Inventory (BDI) scale) over up to six months. There was no significance in the drop-out rates between intervention and comparator groups (RR 0.85, 95\% CI 0.58 to 1.24 ; six studies; $\mathrm{n}=698$ ).

The authors concluded that moderate quality evidence shows that psychotherapy added to usual care is beneficial for depressive symptoms both for response and remission rates over the short term in TRD. It may be beneficial over medium and long term as well. But most evidence was derived from a single large study. At the moment there is no evidence to suggest that switching to psychotherapy is more beneficial for patient with TRD than continuing an antidepressant.

\section{Deep brain stimulation for treatment resistant depression (5)}

Deep brain stimulation (DBS) is another modality being studied for treatment-resistant depression. Blinded, randomized controlled trials of active versus sham treatment have been limited to small numbers.

The authors conducted a systematic review and metaanalysis on the effectiveness of deep brain stimulation (DBS) in depression. Cochrane Central Register of Controlled Trials, PubMed/Medline, Embase and PsycINFO, Chinese Biomedical Literature Service System, and China Knowledge Resource Integrated Database were searched for single- or double placebocontrolled, crossover, and parallel-group trials in which DBS was compared with sham treatment using validated scales.

Ten papers from nine studies met inclusion criteria, all but two of which were double-blinded RCTs. The main outcome was a reduction in depressive symptoms. It was possible to combine data for 190 participants. Patients on active, as opposed to sham, treatment had a significantly higher response $(\mathrm{OR}=5.50 ; 95 \% \mathrm{CI}=2.79$, 10.85; $\mathrm{p}<.0001)$ and reductions in mean depression score $(\mathrm{SMD}=-0.42 ; 95 \% \mathrm{CI}=-0.72,-0.12 ; \mathrm{p}=.006)$.

However, the effect was attenuated on some of the subgroup and sensitivity analyses, and there were no differences for most other outcomes. In addition, 84 participants experienced a total of 131 serious adverse effects, although not all could be directly associated with 
the device or surgery. Publication bias is further limitation to be considered.

DBS may show promise for treatment-resistant depression but remains an experimental treatment until further data are available.

Elevated tumor necrosis factor-alpha receptor subtype 1 and the association with abnormal brain function in treatmentresistant depression (6)

The pathophysiology of treatment resistant depression (TRD) cannot be explained only through the monoamine hypothesis. MDD is known to activate inflammatory response system. Elevated levels of cytokines have been shown to predict treatment resistance.

The authors of this study hypothesized that increased TNF- $\alpha$ R1 is more associated with impaired brain function in TRD patients than non-TRD patients. This study used 18F-fluorodeoxyglucose positron emission tomography (18F-FDG-PET) to investigate glutamatergic neurotransmission in selected brain regions in patients with MDD and healthy control subjects. The 18F-FDG signals were used as a proxy measure to quantify glutamatergic neurotransmission in the human brain.

The study included 14 patients who responded to treatment, 20 patients who were treatment resistant and 34, age, sex and level of education matched healthy controls. Patients with major physical disorder, a history of substance use disorders or major psychiatric comorbidities were excluded.

The severity of the depression was assessed clinically and by using the 17-item Hamilton Rating Scale for depression. The proinflammatory cytokines of all subjects, including C-reactive protein (CRP), interleukin2 receptor (sIL-2R), soluble interleukin-6 receptor (sIL$6 \mathrm{R})$ and TNF- $\alpha$ R1 were assessed by enzyme-linked immunosorbent assays (ELISA).

Results showed that TNF- $\alpha$ R1 level was higher in patients with MDD compared to healthy controls. Among patients with MDD, those with TRD had higher levels of TNF- $\alpha$ R1 compared to patients who responded to treatment. In the depressed group, higher serum concentrations of TNF- $\alpha$ R1 significantly correlated with decreased standardized uptake value in anterior cingulate cortex (ACC) and bilateral caudate nucleus.
Authors acknowledge that the small sample size, not measuring the levels of stress and indirect measuring of glutamatergic transmission as limitations of the study.

In conclusion, increased levels of TNF- $\alpha$ R1 may be associated with impaired glutamatergic neurotransmission of caudate nucleus and ACC in MDD patients, particularly in those with TRD. They also highlight the importance of chronic inflammation in the pathophysiology of TRD and its potential as a mode of treatment.

\section{Declaration of interest}

None declared

D Ariyasinghe, Department of Psychiatry, Faculty of Medicine, University Peradeniya

SS Williams, Department of Psychiatry, Faculty of Medicine, University Kelaniya

Corresponding author: D Ariyasinghe

Email: dewasmika.ariyasinghe@gmail.com

\section{References}

1. Zhou X, Michael KD, Liu Y, Del Giovane C, Qin B, Cohen $\mathrm{D}$, et al. Systematic review of management for treatmentresistant depression in adolescents. BMC Psychiatry 2014; $14: 340$.

2. Miller ML, Luu H, Gaasedelen O, Hahn-Ketter AE, Elmore A, Dezhkam N. Long-Term Cognitive and Psychological Functioning in Post-Electroconvulsive Therapy Patients. J ECT 2018 May 1. [Epub ahead of print]

3. Albott CS, Lim KO, Forbes MK, Erbes C, Tye SJ, Grabowski JG, et al. Efficacy, Safety, and Durability of Repeated Ketamine Infusions for Comorbid Posttraumatic Stress Disorder and Treatment-Resistant Depression. J Clin Psychiatry 2018; 79(3). pii: 17m11634.

4. Ijaz S, Davies P, Williams CJ, Kessler D, Lewis G, Wiles N. Psychological therapies for treatment-resistant depression in adults. Cochrane Database Syst Rev 2018; 5: CD010558.

5. Kisely S, Li A, Warren N, Siskind D. A systematic review and meta-analysis of deep brain stimulation for depression. Depress Anxiety 2018; 5(5): 468-480.

6. Huang MH, Chen MH, Tu PC, Bai YM, Su TP, Yang BH. Elevated tumor necrosis factor-alpha receptor subtype 1 and the association with abnormal brain function in treatment-resistant depression. J Affect Disord 2018; 235: 250-256. 